
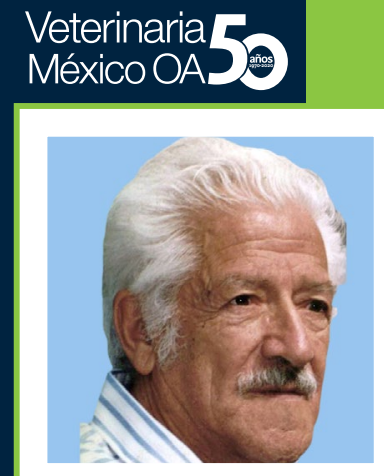

Manuel Ramírez Valenzuela

El Dr. Manuel Ramírez Valenzuela realizó sus estudios profesionales entre 1942-1947 en la Escuela Nacional de Medicina Veterinaria y Zootecnia, y obtuvo el diplomado en Salud Pública Veterinaria en la Escuela de Higiene de la Universidad de Toronto, Canadá en 1955. Ingresó como investigador a la Dirección General de Investigaciones Pecuarias de la Secretaría de Agricultura y Ganadería (SAG), donde se enfocó en toxicología y microbiología diagnóstica. Destacan sus investigaciones epizootiológicas de brotes de Fiebre aftosa, Rabia paralítica bovina y Newcastle. Para esta última enfermedad contribuyó en el aislamiento del virus que posteriormente derivó en una vacuna. La UNAM lo nombró Profesor emérito en 1981.

* Profesor Emérito de la Universidad Nacional Autónoma de México

Jefe del Colegio de Higiene,

Facultad de Medicina Veterinaria y Zootecnia, Universidad Nacional Autónoma de México, México

Ramírez Valenzuela M. El establecimiento de la primera escuela de agricultura y veterinaria en México. Sus antecedentes y primera etapa de vida. Veterinaria México. 1978;9(Suplemento):5-22

Publicado: 2020-09-30

Información y declaraciones adicionales en la página 14

CD Derechos de autor:

Manuel Ramírez Valenzuela 2020

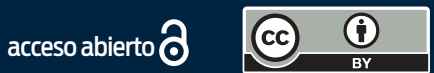

Distribuido bajo una Licencia Creative Commons Atribución 4.0 Internacional (CC-BY 4.0)
Veterinaria

\section{El establecimiento de la primera escuela de agricultura y veterinaria en México. Sus antecedentes y primera etapa de vida}

Manuel Ramírez Valenzuela*

La educación científica en Medicina Veterinaria se inició en Lyon, Francia, ciudad en la que se fundó en 1762 la primera Escuela de Medicina Veterinaria por Claude Borgelat, abogado, mosquetero y escudero del rey Luis XV. Hacia 17501753 publicó un tratado de equitación Elementos de Hipiátrica en donde se indica la necesidad de establecer escuelas especiales para la formación de veterinarios, tiene el apoyo del ministro Bertin y un decreto del Consejo Real establece el 4 de agosto de 1761 la primera Escuela Veterinaria del Mundo, la cual abre sus puertas en Lyon el 16 de febrero de 1762 con sólo dos alumnos hacia 1765 ya tenía 25.

A las Escuelas de Francia "llegan de muchos países de Europa estudiantes extranjeros, que a su vez al regreso, establecerán en sus países Escuelas de Veterinaria semejantes a las francesas". Once Escuelas se establecen en países europeos en el último cuarto del siglo XVIII y diez más en la primera mitad del siglo XIX.

La primera Escuela de Veterinaria que se establece en América es la de México por el decreto del 17 de agosto de 1853, que abriría las clases de Veterinaria en 1856 con siete alumnos, la segunda en Guelph, Canadá, en 1862 y la tercera en 1879 en Ames, Iowa, Estados Unidos de Norteamérica.

En la época prehispánica ya se realizaban prácticas relacionadas con la Medicina Veterinaria, en los mercados de la Gran Tenochtitlán había oficiales que se encargaban de que los alimentos que se vendían estuviesen en buenas condiciones y en las "casas de fieras" de los emperadores aztecas había personal que cuidaba de la salud y alimentación de los animales.

En la Nueva España, el cronista de la conquista y albeitar don Juan Suárez de Peralta sobrino de Hernán Cortés “vezino y natural de México", escribió probablemente hacia 1575-1580, el primer tratado de Medicina Veterinaria escrito en

\section{Cite this as:}

Ramírez Valenzuela M. El establecimiento de la primera escuela de agricultura y veterinaria en México. Sus antecedentes y primera etapa de vida. Veterinaria México OA. 2020:7(3). doi:10.22201/fmvz.24486760e.2020.3.920. 
América el Libro de Aveitería "en el cual se contienen muchos primores tocantes a la albeyteria nunca vistos, ni oydos ni escrito los autores ninguno, moderno ni antiguo: especialmente lo que es curar a los cauallos, y todas las bestias de pata entera por pulso y orina y donde se le hallara el pulso y como se conocerá la orina quando demuestra por ella augmento de sangre y crecimiento de humores, y las colores que demuestra en materia de albeytería, no puestas en práctica ni en theoria, sacado por esperiencia por, don Juan Suarez de Peralta. Laus Deo, Amen".

El manuscrito original se encuentra en la Biblioteca Nacional de Madrid en $4^{\circ}$ con 154 hojas sin numerar L 191, fue paleaografiado por el Dr. Nicanor Armanza Herranz y editado por el Dr. Guillermo Quezada Bravo en 1953 en la Editorial Albeitería en México.

Durante la Colonia la práctica de la Medicina Veterinaria era realizada por albeitares procedentes de la metrópoli; pocos años después de la Conquista según datos que se encuentran en el Archivo de Notarías, los contratos de compra venta de animales se efectuaban "a la vista de albeytar". Posteriormente el ejercicio de los albeitares fue nombrado por las Leyes de Indias.

En este trabajo para conmemorar el 125 aniversario del establecimiento de la Escuela, señalaremos los aspectos más importantes de los antecedentes, su establecimiento y del primer periodo de vida.

\section{Los primeros intentos para establecer la educación superior en agricultura y veterinaria en México}

En la Nueva España y en las primeras décadas del México independiente no existían instituciones de educación superior destinadas para la formación de profesionistas en el área de la conservación y aprovechamiento de los recursos vegetales y animales. En los "Establecimientos" de Ciencias Médicas y de Ciencias Físicas y Matemáticas, fundados después de la clausura de la Universidad Nacional y Pontificia por la iniciativa del Presidente Valentín Gómez Faras y del Dr. Mora, no se contemplaba la enseñanza de la Agricultura y ni de la Veterinaria.

La situación de estas dos actividades en esa época del México independiente era similar a la prevalente en la Colonia, el ilustre sabio mexicano Dr. Leopoldo Río de la Loza, la señala: "Durante los primeros doce años, siguientes a la independencia de México, en 1821, la industria agrícola permaneció estacionaria. Muy raro propietario introdujo algunas modificaciones en el cultivo, algunas reformas en los beneficios de sus productos, y esto luchando como debe suponerse, con un enemigo poderoso, que lo es siempre el de las ciegas costumbres y el de las rutineras preocupaciones. Mas al fin, la introducción y la lectura de buenos libros, los viajes a países extraños, emprendidos por algunos de los propietarios y por otros de nuestros compatriotas amantes de las ciencias; los adelantamientos y aclimatación de ciertos ramos auxiliares de la Agronomía; el uso de alimentos y medicamentos, hasta entonces desconocidos; la introducción y cultivo de nuevas plantas de jardinería, y más aún el entusiasmo patriótico, que alentaba a los promovedores de las mejoras materiales, todo contribuyó a que la industria agrícola 


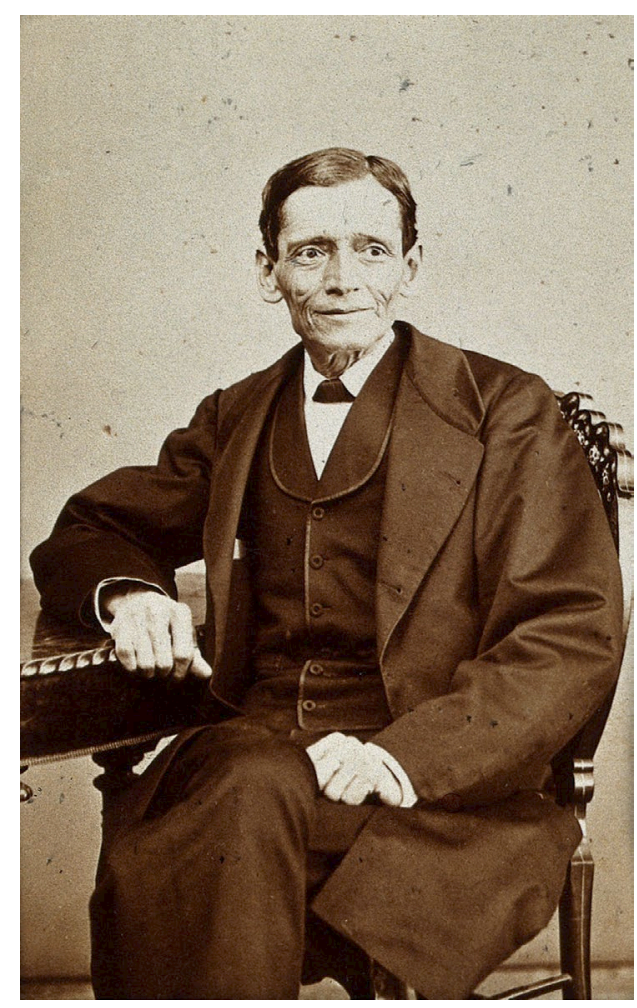

Sr. Prof. Don Leopoldo Río de la Loza

no fuera indiferente a varios particulares y á algunas de las administraciones que han gobernado al país".

En ese período se realizaron varios intentos para establecer la enseñanza de la Agricultura y de la Medicina Veterinaria en México: "en los últimos meses de 1833 apareció un plan general de estudios, por el cual se dispuso entre otras cosas, que en el Hospicio y Huerta de Santo Tomás, se estableciera una cátedra de Botánica, una de Agricultura práctica y otra de Química aplicada a las Artes"; la ley fue derogada sin que se establecieran las cátedras".

En 1835 el presbítero Miguel Guerra donó fondos para "el establecimiento en Guadalajara de una Escuela de Agricultura, cuya benéfica disposición no llegó a realizarse".2 $^{2}$

En 1839, en el decreto de organización del Ejército Nacional, expedido por el Presidente Anastasio Bustamante, en la fracción 18, se indica lo que probablemente es el primer dato relativo a la creación de una Escuela de Veterinaria en México: "Habrá una Escuela Veterinaria que se establecerá en esta capital, con un profesor que gozará el sueldo y consideraciones de Teniente de caballería". ${ }^{3}$

En 1843 el Lic. José Urbano Fonseca estableció en el Gimnasio Mexicano del Olivar del Conde un centro de enseñanza agrícola, el cual se inauguró en septiembre de 1845, sin embargo, "la falta de cooperación por parte de los gobiernos", "los acontecimientos políticos" y el "haber consumido sus fondos propios el patriota desinteresado todo contribuyó a la clausura".

El hombre de letras y distinguido político Lic. Don Lucas Alamán, uno de los más ilustres intelectuales mexicanos de la primera mitad del siglo xix, fue de 
los que más propugnaron por la introducción de la enseñanza agrícola y la tecnificación de la Agricultura y la Ganadería.

En el pensamiento de Lucas Alamán es evidente el interés de establecer escuelas de Agricultura y de Artes y de conducir a la juventud al estudio de otras carreras, "pués en ellas se formarán agricultores y artesanos que conociendo por principio los ramos que ejerzan, estarán en estado de aplicar á ellos los conocimientos teóricos y hacer todos los experimentos necesarios para aprovechar los grandes elementos que la naturaleza ha esparcido en nuestro extenso y variado territorio. En el estado en que han llegado la Agricultura y las Artes, no se puede dar un paso cierto en ellas, ni trabajar con economía y perfección, sin los principios científicos que les sirvan de fundamento". 5

Agregaba: "las carreras de estudios que hoy se presentan a la juventud mexicana, sólo la conducen á aquellas profesiones literarias necesarias para la sociedad, pero en que la sobreabundancia de individuos que las ejerce puede ser peligrosa para ésta. Es menester presentarle otros caminos que conducen a los adelantos positivos y que por no estar todavía trillados, proporcionan más copiosos frutos; es preciso enseñar al propietario rural el arte de sacar mayores rentas de sus bienes, y es menester formar una clase de dependientes de campo instruidos de que hay tanta escasez". ${ }^{6}$

Alamán convencido de la utilidad de establecer esas escuelas y estando a cargo de la Dirección de Industrias, "pidió y promovió su creación durante la administración provisional y mandadas fundar por el decreto de 2 de octubre de 1843, trabajo con solicitud contínua en la adquisición de una hacienda y un edificio á propósito para plantearlas, redacto los reglamentos para ambos establecimientos e hizo formar los programas de estudios, ha solicitado personas capaces de dirigir una enseñanza desconocida hasta ahora entre nosotros"?

El 2 de octubre de 1843, el presidente Antonio López de Santa Anna expidió el decreto en que se indicaba el "establecimiento de las Escuelas de Agricultura y de Artes" 8 y el 13 de abril de 1844 el decreto en que se autorizaba al Gobierno para "la compra de una finca en que se establezca la escuela de Agricultura, y para desponer los gastos que exija dicha escuela y la de artes"."

"Para las lecciones prácticas de Agricultura se compró la Hacienda de la Ascensión en las inmediaciones de esta capital y para el alojamiento de los alumnos y las lecciones teóricas de este ramo así como también para la escuela de Artes se ha hecho la adquisición del Hospicio de San Jacinto, que está contiguo a las tierras de la misma hacienda." ${ }^{10}$ Es esta la primera ocasión en que se hace mención del Hospicio de San Jacinto local que ocuparía por cerca de un siglo hasta 1954 la Escuela de Medicina Veterinaria y hasta 1923 la Escuela de Agricultura.

Como director de la escuela de Agricultura fue designado "Don Melchor Ocampo y de la de Artes el Tte. Coronel Bruno Aguilar" y la apertura de ambas escuelas se efectuaría cuando "se hayan cubierto los adeudos pendientes por las compras de las fincas y se pueda contar con los medios necesarios para su mantenimiento".

Los adeudos no se lograron cubrir, pues los fondos originales se redujeron solamente al $1 \%$ de los derechos de las aduanas de Veracruz y Tampico, pero por 
las interrupciones solamente alcanzaron "más que para el pago de parte del valor de la Hacienda de la Ascensión y del Hospicio de San Jacinto".11

Río de la Loza menciona: "así pasaron más de tres años sin que verificara la apertura de clases, porque disminuidos notablemente los fondos de la Dirección de Industria por el bloqueo y varias otras causas; exhausto el tesoro público y urgido de necesidades más apremiantes; denegado por la Mitra de México el reconocimiento del capital que tenía impuesto en la Hacienda de la Ascensión, y en consecuencia anulada la venta de la finca; invadido más tarde el territorio por los norteamericanos, sin poderse ocupar la nación toda, más que de la guerra á que tan injustamente provocada, los gastos erogados quedaron perdidos, los trabajos comenzados inútiles y todos los sacrificios estériles."12

En 1846 el Gobernador del Estado de México D. José Gómez de la Cortina señalaba en los "Estatutos de la Sociedad de Agricultura del Estado de México, el cuidado de organizar la enseñanza agrícola con el establecimiento de escuelas especiales. Los acontecimientos políticos se opusieron como antes a la realización de este proyecto".12

\section{Establecimiento de la enseñanza agrícola en el Colegio de San Gregorio}

Fundado desde 1574, el Colegio de San Gregorio, después de la consumación de la Independencia fue una institución de los jesuitas dedicada a la enseñanza de las Humanidades y la Jurisprudencia; hacia 1849 el infatigable promotor de la enseñanza agrícola Lic. José Urbano Fonseca, miembro de la Junta Directiva del Colegio "propuso y logró que se aprobara un plan de enseñanza agrícola, cuyos fundamentos consistían, para la teoría el servicio casi gratuito de algunos profesores, y para la práctica, la propiedad rural que tenía dicho Colegio, á saber, la Hacienda de San José Acolman y sus ranchos anexos en el partido de Texcoco, el plan de los programas de cada una de las cátedras se publicaron en 1850 bajo el título de Presente a los Agricultores o Noticia de los ramos de Enseñanza en el Colegio de San Gregorio y en su Hacienda de San José Acolman"13

El Lic. José Urbano Fonseca nació en 1792, fue en 1847 regidor del Ayuntamiento y durante la invasión americana contribuyó al establecimiento del Hospital de San Pablo y de la Escuela de Sordomudos, junto con Río de la Loza y José Fernando Ramírez formó parte de la Junta para la organización de la enseñanza profesional; durante la intervención se negó a formar parte de la asamblea de Notables; tuvo cargos en el Imperio de Maximiliano y por tal motivo estuvo en prisión quedó libre por la amnistía del Presidente Juárez, murió el 21 de junio de 1871.

En el mes de abril de 1850 se dio a conocer en una circular a los gobernadores de los estados el establecimiento de la carrera agrícola en el Colegio de San Gregorio para que "haciéndolo público en el estado á su mando sepan los ciudadanos que tienen ya donde dedicarse a aprender científicamente una carrera tan útil”,14 y en 1851, se aprobó "se dieran quinientos pesos para hacer práctica la enseñanza de la Química Agrícola”. 
En 1852 en el Gobierno del Presidente Mariano Arista, el Lic. José Urbano Fonseca fue designado Ministro de Colonización e Industria y dispuso que ésta "sostuviera en San Gregorio cuatro alumnos destinados precisamente á los estudios agronómicos; más tarde pagó igualmente por un solo año, la colegiatura de un quinto alumno. En todos los jóvenes se notaba en esa época, la decidida inclinación con que habían adoptado y seguían la nueva carrera, llegando el caso de que aún algunos de los que antes de organizar las cátedras habían comenzado las de Jurisprudencia, la abandonaran por la de Agricultura." ${ }^{15}$

\section{Establecimiento del Colegio Nacional de Agricultura con sus escuelas de Agricultura y de Veterinaria}

El año de 1853 el Presidente Antonio López de Santa Anna, expidió el decreto que establecía el Ministerio de Fomento, Colonización, Industria y Comercio encargándose como titular al Ing. Joaquín Velázquez de León.

Menciona Don Francisco Sosa: "ninguna persona más a propósito que el Sr. Velázquez para ser distinguido con tan honroso y difícil cargo, pués bién conocidas eran sus virtudes cívicas, la instrucción facultativa y las prendas morales que le adornaban" ${ }^{16}$

Velázquez de León, Coronel de ingenieros, diplomático, político y catedrático en el Colegio de Minería, había sido "uno de los colaboradores en los proyectos de enseñanza agrícola”.

Correspondían al Ministerio de Fomento, Colonización, Industria y Comercio, la creación de establecimientos de enseñanza en sus ramos y para tal propósito, "el Sr. D. Joaquín Velázquez de León, su sobrino el Sr. D. Miguel, y con dos promovedores de esa reforma importante, se ocuparon desde luego en la formación de la ley". ${ }^{15}$ Es probable que los dos promovedores a que se refiere Río de la Loza fueran, el Lic. Lucas Alamán como Secretario de Gobernación y el Lic. José Urbano Fonseca miembro de la Junta Directiva del Colegio de San Gregorio y que intervino en la elaboración del plan de estudios de la carrera de Veterinaria el Dr. Eugenio Bergeyre Veterinario militar francés, egresado de la Escuela Nacional Veterinaria de Tolosa y que en 1853 había sido contratado por el Gobierno de Santa Anna "teniendo especialmente a su cargo las caballerizas del Presidente". ${ }^{17}$

Finalmente el decreto fue expedido por el Presidente Antonio López de Santa Anna el 17 de agosto de $1853 .{ }^{18}$ En el Art. $1^{\circ}$ Se indica: "Se establece una Escuela de Veterinaria, agregada a la de Agricultura que existe en el Colegio Nacional de San Gregorio; en el 20 "ambas Escuelas llevarán el nombre de Colegio Nacional de Agricultura" para su sostenimiento se le asignaron: "el sobrante de todos los bienes de parcialidades, después de cubiertos los gastos á que estén afectos legalmente" que corresponden a cada año de estudios, en el correspondiente al $5^{\circ}$ se indican: "I. Lección diaria de Veterinaria Elemental y III. Práctica de Veterinaria".

En el Art. 15, se refiere a la planta de personal y sueldo; el de un catedrático de Veterinaria ascendía a 800 pesos anuales. 
En el Art. 20 se indica la dependencia del Colegio de Ministerio de Fomento, Colonización, Industria y Comercio y en el 21 la supresión de las cátedras en el Colegio de San Gregorio, con la excepción de las de Agricultura.

La Escuela de Agricultura, además del Colegio de San Gregorio que estaba situado en lo que en la actualidad corresponde a la Secundaria No. 6 en las calles de San Ildefonso, contaba con los terrenos de la Hacienda de Acolman, la cual "se hallaba a mayor distancia de lo que conviene á la sobrevigilancia, auxilios y conveniencias, tanto didácticas como familiares, y sabiendo en fin que privado el Gobierno de la Hacienda de la Ascensión, sólo contaba con el edificio de San Jacinto, y no con sus terrenos, se propuso y logró que comprara éstos el Gobierno"19

Al edificio de San Jacinto, situado a lo que hoy corresponde a la Calzada México Tacuba esquina con la calle del Maestro Rural; se "trasladaron los muebles y capitales que se habían agregado al primitivo Colegio de San Gregorio". "La biblioteca se trasladó a San Jacinto sin conceder ni la más pequeña de las mesas giratorias o atriles, ni una obra de Buffon que se hallaba duplicada. Llevose igualmente todo lo útil y valioso, como sillas finas, un clave, mesas, bancas, pinturas, instrumentos, muestra de dibujo y de escritura, cocina, vidrieras, museo de antigüedades y otras cosas más menudas. Por último, se mandó que para la fábrica material del Colegio de Agricultura, se entregaran 500 pesos que el Colegio de San Gregorio debía de haber gastado en sus premios".

En septiembre de 1853 se restableció en México la Compañía de Jesús y .... "dispuesto que se volviera a encargar de la iglesia de Loreto y de la dirección de la juventud, como antes lo estuvo, le fueron entregados ambos edificios, y sólo se consiguió dividir, según convenio, algunos de los muebles, utensilios y libros que pasaron a San Jacinto; que fueran a él los alumnos que quisieron seguir la carrera agrícola... 19

Indica Río de la Loza que Velázquez de León ...juzgó que sería más conveniente el confiar la enseñanza y dirección agrícolas, a un profesor traído de Europa; más como al llegar el profesor, á quien se había contratado por cinco años, no sólo se vio que desconocía el idioma español, sino también las costumbres, las influencias climatológicas y demás exigencias agronómica, pasaron dos años sin que los alumnos lograran hacer las debidas aplicaciones de los estudios preparatorios que ya tenían, ni menos adquirir los profesionales, de que más necesitaban"...

El 7 de octubre de 1853 un decreto de Santa Anna estableció un impuesto sobre frutos y efectos extranjeros que fueran introducidos a la capital de ..."un real por cada bulto de media carga de mula”... aplicable a ..."la conservación y Fomento de la Escuela de Agricultura en la parte que no le basten sus propios recursos"... ${ }^{21}$

Finalmente los alumnos de Agricultura pasaron a San Jacinto y ..."tomaron posesión del nuevo plantel el 22 de febrero de 1854”... El Lic. José G. Arriola quien funcionaba entonces como Rector del Colegio de San Gregorio "siguió en San Jacinto con el mismo encargo; las cátedras comenzaron y al concluir el año de 1855, se verificaron los exámenes de las clases preparatorias"... ${ }^{22}$

"En cuanto a la Veterinaria, nada se hizo, no obstante hallarse organizada la carrera en la citada ley". 


\section{La Escuela Nacional de Agricultura y Veterinaria al triunfo del Plan de Ayutla. Modificación del plan de estudios. Se inician las cátedras de veterinaria}

El 1 1 de marzo de 1854 fue proclamado el Plan de Ayutla, y al triunfo de la Revolución fue derrocado el Presidente Santa Anna el 9 de agosto de 1855; después del Gobierno provisional del Gral. Juan Alvarez se hizo cargo del poder ejecutivo Don Ignacio Comonfort quien designó como Secretario de Fomento al Sr. Manuel Siliceo y como Oficial Mayor a Don Manuel Orozco y Berra. Menciona Río de la Loza que: ..."algunas de las personas que habían trabajado en las anteriores empresas, fueron nombrados desde luego, para modificar ó mejor dicho, mejorar la ley de $1853{ }^{\prime \prime . .22}$

El 4 de enero de 1856, se expidió el decreto por el Presidente Comonfort que modificaba la enseñanza en la Escuela Nacional de Agricultura; ${ }^{23}$ en el Art. $1^{\circ}$ se indicaba la división de la enseñanza agrícola en: "superior, para formar "administradores instruidos" y "común” para formar "mayordomos inteligentes". En el Art. $4^{\circ}$ se señalaba una duración de cinco años para la enseñanza agrícola superior y de tres para la común y las asignaturas correspondientes a cada año, en el $4^{\circ}$ año se indicaba "un curso elemental y completo de Veterinaria de lección alternada".

A los alumnos aprobados en los cinco años de la carrera se les extendía el "Título de Agricultor teórico práctico" y los que aspiraban al título de "Profesor de Agricultura" debía de cursar además "dos años las cátedras de perfección”.

Los alumnos que pretendían únicamente asistir a los cursos de Veterinaria y al de Operaciones y prácticas de Herrajes, se les exigía "como únicos estudios preparatorios, los que se designan para los agricultores en el primer año”... A los alumnos que concluían la carrera se les extendía ...el certificado de Mariscales y después de tres años de publicada esta ley, sólo ellos podrán dirigir bancos públicos de albeitería. Los actuales propietarios de éstos tienen la obligación de matricularse en el Colegio, para continuar en ellos"...

En el Art. 13 se menciona la forma de elección del Director: ..."que será uno de los catedráticos, propuesto por ellos mismos, renovado cada cinco años y nombrado por el supremo Gobierno"...

En los Arts. 16, 17 se menciona "un cuerpo de profesores sustitutos" y la creación de "la Junta de Catedráticos y en el Art. 22 el nombramiento por el Gobierno de una "Junta protectora del establecimiento y enseñanza de la Agricultura", siendo sus principales atribuciones: "el exacto cumplimiento de esta ley y de los reglamentos, y proponer al mismo Gobierno las mejoras y adelantos de que sea susceptible el Colegio".

En enero de 1856, los profesores de la Escuela propusieron al Dr. Leopoldo Río de la Loza como Director, siendo nombrado por el Gobierno para tal cargo recibiendo del Lic. José Guadalupe Arriola Rector que fue del Colegio de San Gregorio y que tenía el mismo carácter en la Escuela de Agricultura en San Jacinto. El Dr. Río de la Loza, nació en la ciudad de México el 13 de noviembre de 1807, ingresó al Colegio de San Ildefonso, estudió Química en el Colegio de Minería y en 1827 recibió el título de Cirujano, en 1828 se recibió de farmacéutico y de Médico Cirujano en 1833; Catedrático de Química desde 1844 en la Escuela Nacio- 
nal de Medicina y en el Colegio de Minería; en 1845 fue nombrado catedrático de Química aplicada a las Artes y a la Agricultura en el Gimnasio Mexicano, en 1852 en el Colegio de San Gregorio y posteriormente en la Escuela de Agricultura en San Jacinto. En 1838 fue miembro de la Primera Academia de Medicina y en 1851 Presidente de la segunda; hasta 1856 había publicado 19 trabajos sobre Química, Farmacia y Farmacología.

El Dr. Río de la Loza, primer Director que tuvo la Escuela, "solicitó del Gobierno que se dieran los recursos necesarios a fin de emprender algunas obras indispensables". El Gobierno del Presidente Comonfort... "no sólo concedió en los días tranquilos lo que le fue pedido, sino que aún en los momentos más críticos porque paso después, lejos de escasear los recursos pecuniarios a la atención personal que le diera al principio, se dedico con el mismo empeño á fomentar la Escuela como sí el Gobierno se hallara en perfecta paz y abundante de recursos" ${ }^{24}$

A un problema que es también frecuente en nuestra época tuvo que enfrentar desde el principio de su gestión el Dr. Río de la Loza, la falta de catedráticos, al respecto menciona: "la dificultad de hállar profesores para la enseñanza de la parte agrícola propiamente dicha ha sido el obstáculo más poderoso con que se ha tenido que luchar".

Otro problema era el de la falta de capacidad didáctica, refiriéndose a una persona que había sido contratada en París, señala ...'falta de método en el catedrático nombrado y por otra parte veía la confianza que se tuvo en él, fundada sin duda, en la estimación que generalmente se ha dado a los extranjeros, como que este había presentado como garantía el título de profesor de Agricultura”... comisionado este catedrático fuera de la Escuela, fue reemplazado por el substituto pero ..."muy poco se adelanto con el cambio, á pesar de que el nuevo nombrado llevaba como su anterior, el título de profesor de agricultura expedido por una de las escuelas europeas; su falta de conocimientos climatológicos, de la aptitud de los trabajadores, de los usos y costumbres del país, y algunos defectos perniciosos a la buena educación y extraños al profesorado, nulificaron la enseñanza y obligaron a la Dirección a que fuera separado"... ${ }^{25}$

Finalmente el Dr. Rio de la Loza resolvió la situación nombrando "a un mexicano de quien se habían hecho muy especiales recomendaciones, como práctico, inteligente y conocedor, no sólo de la Agricultura del país, sino también de la de Norteamérica, á donde había sido educado. Preciso es decir que los alumnos hicieron indudablemente más que antes, se dio algún orden a los cursos, y auxiliado el profesor con el servicio gratuito de la clase de primer año de Agricultura, de la cual se encargó el Director en enero de 1860, comenzaron las excursiones agrícolas, y con ellas a mejorarse la enseñanza". ${ }^{26}$

En lo que respecta a la enseñanza de la Medicina Veterinaria el Dr. Río de la Loza no tuvo problemas, pues las cátedras estaban a cargo del competente veterinario militar francés el Dr. Eugenio Bergeyre, quien radicaba en México desde 1853.

El Dr. Bergeyre tuvo a su cargo las cátedras de Delineación, Anatomía y Farmacología, Mariscalía y Exterior, Patología y Cirugía Veterinaria y de Operaciones y Herrajes en la Escuela de Agricultura y Veterinaria, siendo por lo tanto 
el primer Médico Veterinario que tuvo la Escuela como profesor y que formó científica y moralmente a las primeras generaciones de Médicos Veterinarios que constituirían los cimientos de la profesión en México.

El profesor de Anatomía y Fisiología era el ilustre Médico Cirujano Ignacio Alvarado, siendo estudiante ganó un concurso para el puesto de prosector de Anatomía en la Escuela de Medicina y desde 1856 era profesor en la Escuela de Agricultura y Veterinaria y "debe haberlo hecho con lucidez puesto que uno de los alumnos más asiduos que tuvo en ella, fue nada menos que don Gabino Barreda". ${ }^{27}$

El 31 de diciembre de 1856, el Presidente Comonfort, expidió una ley en la que se organizaban los estudios de Agricultura, Veterinaria e Ingeniería dentro de la Escuela Nacional de Agricultura; ya que en esta "se hallaban establecidas varias de las cátedras comunes, con las que corresponden á los estudios de los ingenieros topógrafos, de los mecánicos y de los civiles". ${ }^{26}$

En el Art. $1^{\circ}$ se indicaba: "En la Escuela Nacional de Agricultura se aumentaran las cátedras necesarias y los medios materiales de enseñanza para que desde luego queden establecidas las carreras de Agricultura, de Veterinaria y de Ingeniería"; en el Art. $6^{\circ}$ se señalaba que: "La enseñanza veterinaria comprenderá la de los Profesores Veterinarios y la de los Mariscales; en el Art $7^{\circ}$ se indicaban "Los Mariscales estudiaran en tres años los ramos que a continuación se expresan: Anatomía General y Descriptiva, Fisiología e Higiene, Exteriores de los Animales Domésticos sus bellezas y defectos, Patología General Externa e Interna, Medicina Operatoria, Práctica de Herrajes y Farmacología", y en el Art. $8^{\circ}$ se indicaba: "Los profesores de Veterinaria estudiaran en cinco años los ramos anteriores y los siguientes: Dibujo Natural y Anatómico, Francés, Inglés y Alemán, Primer Curso de Matemáticas, Física y Química".28

Esta ley afectó en cierta forma a la Escuela, pues "pronto se vio como estaba previsto, que varios de los alumnos prefirieron seguir estas carreras, especialmente la de Topógrafos, y que para tener alumnos que se dedicaran a la de Veterinaria, fue necesario solicitarlos empeñosamente, lográndose con no pocas dificultades, que se presentaran siete de la Escuela de Artes, establecida en 1857". 29

Los alumnos a los que se refiere el Dr. Río de la Loza fueron: Narciso Aguirre, Ignacio Salazar, José de la Luz Gómez, José E. Mota, José María Lugo, Manuel G. Aragón y Mariano G. Aragón.

Después de vencer tantas dificultades al fin se logró abrir "el 9 de abril de 1858 bajo la dirección del Dr. Ignacio Alvarado, el primer curso formal de Veterinaria que se dio en la Escuela".

En el periodo de 1859 a 1860, que comprende los pronunciamientos contra el Gobierno de Comonfort y la Revolución de Reforma, los fondos de la Escuela fueron dispuestos para otros fines diferentes al de la educación, "en cambiar a los miserables en acaudalados y lo que es peor, en regar el extenso territorio mexicano con la sangre de sus hijos". 29

En 1858 ascendían a 859,776 pesos los bienes de la Escuela que "desaparecieron del modo siguiente: en febrero de 1859, entregados al Gobierno del General Miramón \$ 264,601. En mayo de 1860, entregados a la misma adminisración \$325,696 y en 1861 á la administración del Sr. Juárez \$269,479”.31 
"En el transcurso de ese periodo en que faltaron los fondos propios, los profesores dejaron de contar con el pago puntual de sus sueldos. Los alumnos no fueron debidamente atendidos en su ropa, calzado y demás necesidades; las obras materiales quedaron suspensas, las labores mal atendidas".

Además a consecuencia de la situación imperante, la Escuela en ocasiones transformada en cuartel tuvo que "trasladar a sus alumnos una vez al Colegio de San Ildefonso y dos al de Letrán". Pero a pesar esta grave situación, "ni un solo año dejaron de verificarse los exámenes finales ni los premios consiguientes".

El 11 de enero de 1861 el Presidente Juárez entró a la capital de la República y en los primeros días del mes de febrero del mismo año era "separado, sin causa alguna, el Director de la Escuela de Agricultura y Veterinaria"; Escobar, considera como causa probable de la separación del Dr. Río de la Loza de la Dirección de la escuela, "la de haber tenido ciertas creencias religiosas". ${ }^{32}$

El Dr. Gabino Barreda, refiriéndose a la actividad del Dr. Leopoldo Río de la Loza como Director de la Escuela señala: “desplegó todas sus dotes económico administrativas en una forma que pudiéramos llamar ideal, todo en la Escuela que se puso a su cargo, llegó a estar arreglado con intachable precisión, los estudios prácticos como los teóricos; los trabajos serios como las distracciones, todo estuvo allí determinando con el cronómetro en la mano".33

Es posible que los resultados de la obra de Río de la Loza en la Escuela y su proyección al medio rural no tuvieron una aplicación efectiva por la falta de lo que en la actualidad conocemos como extensión agropecuaria, esto lo indica Barreda cuando señala: "Si a Río de la Loza se hubiese encomendado la importante misión de ilustrar a los propietarios, por todos los medios posibles y a cualquier costa, sea por medios de publicaciones, sea mandando a sus profesores a hacer experimentos a las fincas de campo de los particulares, a costa del Erario, para demostrar las ventajas de tales o cuales operaciones científicamente dirigidas; sea ejecutándolas en el establecimiento en presencia de esos mismos propietarios; sea por cualesquiera otros arbitrios que él habría sabido imaginar; si se le hubiera encargado, en fin, crear la necesidad antes de satisfacerla, preparar la demanda antes de la oferta, curar la inapetencia antes de presentar los manjares, la incansable actividad de nuestro héroe y sus inapreciables dotes prácticas habrían asegurado un éxito completo. Pero por una fatal, aunque disculpable ilusión, se le sacrificó estérilmente en la infructuosa tarea de formar excelentes músicos en un pueblo de sordos. Tanta actividad y tanta ciencia no lograron mejorar nuestra Agricultura. En su entusiasmo por servir en cuanto se le creía útil, Río de la Loza se dejó llevar a una empresa cuyas consecuencias no eran sin duda, tan fáciles de prever como lo son de aplicar ahora que ya se realizaron".

Sin embargo es el propio Río de la Loza que rechaza particularmente en lo que se refiere a Veterinaria a lo señalado por Barreda: "no puede dudarse que fue una mejora de grande importancia para el país. Con tal determinación se creó una nueva carrera, se procuró destruir el charlatanismo y la perniciosa ignorancia de los antiguos albéitares, se proporcionaron al público médicos veterinarios inteligentes y moralizados, a quienes pudiera confiar los animales enfermos, que además de tener un valor real, tienen por lo común el estimativo que es incal- 
culable; en fin con el sólo hecho de organizar esa carrera se demostró que los mexicanos no desconocían las exigencias sociales de los países ilustrados, ni la de procurarlas al suyo".34

\section{La escuela durante la dirección del Dr. Juan $\mathbf{N}$. Navarro Modificación del Plan de Estudios. Egresan los primeros veterinarios}

En febrero de 1861 sucede en la Dirección de la Escuela al Dr. Leopoldo Río de la Loza el Dr. Juan N. Navarro; originario de Michoacán, fue profesor de Clínica Externa en la Escuela Nacional de Medicina en el período de 1848 a 1860, en 1847 luchó contra los americanos en Churubusco y fue hecho prisionero. En 1863 como Jefe del Cuerpo Militar cayó prisionero en el sitio de Puebla y logró escapar; el Presidente Juárez lo nombró Cónsul de México en Nueva York.

El 15 de abril de 1861 el Presidente Juárez expidió "La Ley sobre instrucción pública en los establecimientos que dependen del Gobierno”, en el Art. 27 se indicaba "Los estudios en la Escuela Especial de Agricultura duraran siete años". En el 1ㅜ, Matemáticas e idioma Francés; en 2º Mecánica, Geodesia y Francés; $3^{\circ}$, Química Aplicada a la Agricultura, Botánica, Dibujo e Inglés; $4^{\circ}$, Teoría de las construcciones rurales, Dibujo e Inglés $5^{\circ}, 6^{\circ}$ y $7^{\circ}$, Agricultura teórico práctica y economía rural; en el $7^{\circ}$ año se enseñará también la Veterinaria teórico práctica. En los últimos cuatro años los catedráticos harán que sus alumnos reciban las lecciones prácticas que consideren necesarias "para su adelantamiento".35

Este plan general de estudios, "fijó algunas bases generales que dicen poco; más como no llegó a observarse, y como las órdenes y disposiciones oficiales eran frecuentes y aún contrarias, debían alterar el orden con perjuicio de la buena instrucción". ${ }^{36}$

El Dr. Navarro era un liberal prominente y como señala Río de la Loza "una persona demasiado influyente en el partido triunfante, se creyó con fundamento, que la Escuela ganaría, recobrando, cuando menos sus bienes ú otros equivalentes. Por desgracia no fue así, pues los ingresos bastaron para cubrir los buenos sueldos de algunos de los empleados, sin que se hiciera en toda esa época adquisición alguna de importancia, ni mejora material, ó se atendiera tal vez el pago puntual de los profesores y a las necesidades de los alumnos". ${ }^{31}$

La ley del 23 de febrero de 1861, había establecido la Secretaría de Justicia e Instrucción Pública y se había designado como titular a Ignacio Ramírez "El Nigromante" quien no tardó en aplicar a una dependencia del Gobierno las normas de la reforma: "olvidaba la Junta Protectora, suprimida la cátedra de Religión, después destituido el capellán y por último cerrada y abandonada la capilla y en libertad los alumnos para seguir la creencia que mejor les pareciera”.

Otros inconvenientes afectaron a la actividad educativa de la Escuela "la música, la gimnasia, el manejo de armas, los idiomas se quitaron; el reglamento quedó sin observancia y las disposiciones económicas constantemente expuestas a las consecuencias de los acuerdos eventuales". 
A principios de 1861, "la Dirección comisionó á dos profesores para que extendieran un proyecto de reforma, este trabajo fue presentado, pero inútil; porque se adoptaron otras modificaciones más o menos notables".

Menciona Flores que "al fin, debido al empeño de los primeros profesores que la inauguraron, los Sres. Alvarado Río de la Loza, Varela, Jiménez (L), Zepeda, Parra y Bergeyre, en 1862 se obtuvieron las primicias, habiéndose recibido los primeros cinco Médico Veterinario"30 que fueron: José de la Luz Gómez, José E. Mota, José María Lugo, Manuel G. Aragón y Mariano G. Aragón.

En 1862 México sufrió la invasión de las fuerzas francesas y en mayo de 1863 después de la caída de Puebla asediaban la capital.

"A principios de 1863, fueron aumentados los sueldos de algunos profesores de Veterinaria, aumentando igualmente el trabajo al de Botánica, con notables inconvenientes, encargándole la clase de Geología y de Mineralogía; establecida y provista una de Zootecnia, reducida a sólo una de las tres de Agricultura, y destituidos por los mismos, dos de los profesores que las servían".

"Cuando las personas que figuraron en el Gobierno de esa época, se decidieron a no defenderse en la capital de la invasión que se aproximaba, dictaron algunas disposiciones generales relativas a los establecimientos Generales de Enseñanza y cuyo objeto final parece que fue su destrucción completa, esto a lo menos puede inferirse observando lo que pasó en la Escuela de Agricultura. Fue arrendada ó acaso se simuló un arrendamiento de la finca por diez años, a favor de la persona que entonces funcionaba como catedrático de Agricultura, pero esto se hizo de tal modo, que desde luego alejo toda idea de realidad. Por otra parte, el ganado vacuno fue vendido, así como los bueyes, bestias de tiro, el coche, alfombras, floreros, objetos de iglesia. Varios de los instrumentos y aparatos fueron extraídos del Colegio y conducidos a casas de particulares, tal vez para asegurarlos". ${ }^{37}$

A pesar de las dificultades en la vida de la Escuela que han sido señaladas por Río de la Loza, determinadas por la lucha social y política y el intervencionismo imperialista de Napoleón III, el balance final de este crítico periodo fue positivo, se estableció la Escuela de Agricultura y Veterinaria y en ella se forjaron las primeras generaciones de Agrónomos y Veterinarios los que a su vez continuarían la obra de sus profesores, unos en las aulas y laboratorios, otros aplicando sus conocimientos a la solución de los problemas del campo y de la Salud Pública.

La mayoría de los profesores, tuvieron que luchar contra la tradición colonial, opuesta a la introducción de una enseñanza nueva y su aplicación al campo, enseñanza que tuvo su origen en las Escuelas de Agricultura y Veterinaria de Francia, fuente constante de conocimientos que persistiría en el curso del siglo xIX y principios del actual. 


\section{Referencias}

1. Río de la Loza L. La agricultura y la veterinaria en la nación mexicana. En: Noriega JM, compilador. Escritos de Leopoldo Río de la Loza, México: Escalante; 1911. p. 284.

2. Río de la Loza L. La agricultura y la veterinaria en la nación mexicana. En: Noriega JM, compilador. Escritos de Leopoldo Río de la Loza, México: Escalante; 1911. p. 285.

3. Decreto expedido por el Gobierno Supremo para organizar los cuerpos de Infantería y Caballería del Ejército Nacional Mexicano, Imp. del Aguila; 1839. p. 4.

4. Río de la Loza L. La agricultura y la veterinaria en la nación mexicana. En: Noriega JM, compilador. Escritos de Leopoldo Río de la Loza, México: Escalante; 1911. p. 285-6.

5. Alamán L. Obras de D. Lucas Alamán, Documentos diversos y muy raros. México: Jus; 1945. p. 302-3.

6. Alamán L. Obras de D. Lucas Alamán, Documentos diversos y muy raros. México: Jus; 1945. p. 307.

7. Alamán L. Obras de D. Lucas Alamán, Documentos diversos y muy raros. México: Jus; 1945. p. 303.

8. Dublán y Lozano. Legislación Mexicana, México: Imp. del Comercio; 1876 T. IV. p. 610-4.

9. Dublán y Lozano. Legislación Mexicana, México: Imp. del Comercio; 1876 T. IV. p. 753.

10. Alamán L. Obras de D. Lucas Alamán, Documentos diversos y muy raros. México: Jus; 1945. p. 304.

11. Alamán L. Obras de D. Lucas Alamán, Documentos diversos y muy raros. México: Jus; 1945. p. 306.

12. Río de la Loza L. La agricultura y la veterinaria en la nación mexicana. En: Noriega JM, compilador. Escritos de Leopoldo Río de la Loza, México: Escalante; 1911. p. 287.

13. Río de la Loza L. La agricultura y la veterinaria en la nación mexicana. En: Noriega JM, compilador. Escritos de Leopoldo Río de la Loza, México: Escalante; 1911. p. 288.

14. Dublán y Lozano, Legislación Mexicana, México: Imp. del Comercio; 1876 T. IV. p. 691.

15. Río de la Loza L. La agricultura y la veterinaria en la nación mexicana. En: Noriega JM, compilador. Escritos de Leopoldo Río de la Loza, México: Escalante; 1911. p. 289.

16. Sosa F. Biografías de mexicanos distinguidos. México: Of. Tip. de la Sría. de Fomento; 1884. p. 1059.

17. Flores AF. Historia de la medicina en México. México: Of. Tip. de la Sría. de Fomento; 1886. T. III. p. 231.

18. Dublán y Lozano, Legislación Mexicana, México: Imp. del Comercio; $1876 \mathrm{~T}$. IV. p. 642-6. 
19. Río de la Loza L. La agricultura y la veterinaria en la nación mexicana. En: Noriega JM, compilador. Escritos de Leopoldo Río de la Loza, México: Escalante; 1911. p. 290.

20. Decorme G. Historia de la Compañía de Jesús en la República Mexicana durante el siglo xix, Guadalajara: Tip. y Enc. Yguiniz, Suc; 1921. T. II. p. 59.

21. Dublán y Lozano, Legislación Mexicana, México: Imp. del Comercio; 1877. T. IV. p. 707-8.

22. Río de la Loza L. La agricultura y la veterinaria en la nación mexicana. En: Noriega JM, compilador. Escritos de Leopoldo Río de la Loza, México: Escalante; 1911. p. 291.

23. Legislación Mexicana, Tomo que comprende de enero a junio de 1856 , México, Imp. de J. R. Navarro; 1856. p. 18-25.

24. Río de la Loza L. La agricultura y la veterinaria en la nación mexicana. En: Noriega JM, compilador. Escritos de Leopoldo Río de la Loza, México: Escalante; 1911. p. 292.

25. Río de la Loza L. La agricultura y la veterinaria en la nación mexicana. En: Noriega JM, compilador. Escritos de Leopoldo Río de la Loza, México: Escalante; 1911. p. 293.

26. Río de la Loza L. La agricultura y la veterinaria en la nación mexicana. En: Noriega JM, compilador. Escritos de Leopoldo Río de la Loza, México: Escalante; 1911. p. 294.

27. Izquierdo JJ. Balance cuatricentenario de la fisiología en México. México: Ed. Ciencia; 1934. p. 172.

28. Dublán y Lozano. Legislación Mexicana, México: Imp. del Comercio; 1877. T. VIII. p. 9.

29. Río de la Loza L. La agricultura y la veterinaria en la nación mexicana. En: Noriega JM, compilador. Escritos de Leopoldo Río de la Loza, México: Escalante; 1911. p. 295.

30. Flores AF. Historia de la medicina en México. México: Of. Tip. de la Sría. de Fomento; 1886. T. III. p. 234.

31. Río de la Loza L. La agricultura y la veterinaria en la nación mexicana. En: Noriega JM, compilador. Escritos de Leopoldo Río de la Loza, México: Escalante; 1911. p. 296.

32. Escobar R. La instrucción agrícola en México. IV Congreso Científico.

33. Discurso pronunciado por el Dr. Gabino Barreda, como representante de la "Asociación de Sociedades Científicas de México", en la apoteosis de Don Leopoldo Río de la Loza, celebrada en la ciudad de México el 15 de noviembre de 1 877, reproducido en Lemoine E. La Escuela Nacional Preparatoria en el período de Gabino Barreda 1876-1878. México: UNAM; 1970.

34. Río de la Loza L. La agricultura y la veterinaria en la nación mexicana. En: Noriega JM, compilador. Escritos de Leopoldo Río de la Loza, México: Escalante; 1911. p. 303.

35. Código de la Reforma, México: Imprenta Literaria; 1861. p. 247. 
36. Río de la Loza L. La agricultura y la veterinaria en la nación mexicana. En: Noriega JM, compilador. Escritos de Leopoldo Río de la Loza, México: Escalante; 1911. p. 297.

37. Río de la Loza L. La agricultura y la veterinaria en la nación mexicana. En: Noriega JM, compilador. Escritos de Leopoldo Río de la Loza, México: Escalante; 1911. p. 299. 